\title{
Laser Repair of Superalloy Single Crystals with Varying Substrate Orientations
}

\author{
S. MOKADEM, C. BEZENÇON, A. HAUERT, A. JACOT, and W. KURZ
}

The casting and repair of single-crystal gas turbine blades require specific solidification conditions that prevent the formation of new grains, equiaxed or columnar, ahead of the epitaxial columnar dendrites. These conditions are best determined by microstructure modeling. Present day analytical models of the columnar-to-equiaxed transition (CET) relate the microstructure to local solidification conditions (temperature gradient and interface velocity) without taking into account the effects of (1) a preferred growth direction of the columnar dendrites and (2) a growth competition between columnar grains of different orientations. In this article, the influence of these effects on the grain structure of nickel-base superalloy single crystals, which have been resolidified after laser treatment or directionally cast, is determined by experiment and by analytical and numerical modeling. It is shown that two effects arise for the case of a nonzero angle between the local heat flux direction and the preferred dendrite growth axis: (1) the regime of equiaxed growth is extended and (2) a loss of the crystal orientation of the substrate often occurs by growth competition of columnar grains leading to an "oriented-to-misoriented transition" (OMT). The results are essential for the definition of the single-crystal processing window and are important for the service life extension of expensive components in land-based or aircraft gas turbines.

\section{INTRODUCTION}

DUE to their outstanding high-temperature properties, nickel-base superalloys are widely used for hot section components in gas turbines. To obtain longer service life of aircraft engines or land-based energy generation systems, single crystals are used for the most solicited highpressure/high-temperature turbine blades. The high cost of these components makes the repair of damaged blades highly desirable, using special welding, brazing, or deposition processes. In all of these processes, it is essential that the single-crystal microstructure is retained and that hot cracks are avoided. Although the concepts presented here are useful for both single-crystal casting and repair, this article will concentrate mainly on repair aspects. Results presented will provide a sound basis for upscaling to an industrial process.

During the last decade, it has been shown that singlecrystal repair is feasible by epitaxial laser metal forming. In this process, alloy powder of the same ${ }^{[1-4]}$ or of different composition to that of the substrate ${ }^{[5,6]}$ is injected into the molten pool formed by a moving high intensity laser beam (Figure 1). By depositing successive layers on top of each other, single-crystal parts such as walls or of more complex geometries have been built onto a single-crystal substrate

S. MOKADEM, formerly Ph.D. Student, Institute of Materials, Ecole Polytechnique Fédérale de Lausanne, 1015 Lausanne EPFL, Switzerland, is with Siemens Power Generation, D-45473 Mülheim a.d. Ruhr, Germany. C. BEZENÇON, formerly Postdoctoral Researcher, Institute of Materials, Ecole Polytechnique Fédérale de Lausanne, is with Novelis Switzerland SA, CH-3960 Sierre, Switzerland. A. HAUERT, Ph.D. A. JACOT, Research Associate, and W. KURZ, Professor, are with the Institute of Materials, Ecole Polytechnique Fédérale de Lausanne. Contact e-mail: alain.jacot@epfl.ch

This article is based on a presentation made in the symposium entitled "Solidification Modeling and Microstructure Formation: In Honor of Prof. John Hunt," which occurred March 13-15, 2006, during the TMS Spring Meeting in San Antonio, Texas, under the auspices of the TMS Materials Processing and Manufacturing Division, Solidification Committee. with a sound and well-bonded interface. This technique is undergoing development and, in the near future, will be used for repair engineering or rapid prototyping.

The processing conditions of the single-crystal repair process must be kept inside a relatively narrow window, which is a characteristic of the alloy. This ensures the epitaxial columnar solidification of the deposited material in the form of a single crystal. The determination of the processing window cannot be achieved efficiently without modeling both the temperature field of the process and the solidification behavior of the alloy. The solidification microstructure (single crystal or polycrystal) can thus be related to the laser processing conditions (such as laser power, scanning speed, beam diameter, etc.).

By a knowledge of alloy properties and using relevant solidification models, a microstructure selection map can be constructed, which shows the structures that will develop as a function of the temperature gradient, $G$, and the growth rate, $V$, which are both imposed by the process. ${ }^{[7]}$ Gäumann et al., following Hunt, derived a criterion for the columnarto-equiaxed transition (CET) of alloys under conditions of laser treatment, i.e., high-temperature gradients and interface velocities. ${ }^{\left[{ }^{[, 9]}\right.}$ Using this model, it was demonstrated that a fully columnar structure is obtained when the ratio $G^{n} / V$ is above a critical value (the exponent, $n$, depending on the alloy). ${ }^{[3]}$ Similar research on superalloy singlecrystal welding has been undertaken by Vitek et al., where the mechanism of stray grain formation during welding of nickel-base single-crystal superalloys was examined and analyzed as a function of composition and welding

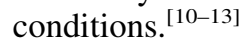

The theoretical background used in these early approaches was limited to two simplifying conditions: (1) the heat flow was collinear with the preferred dendrite growth orientation ([001] for cubic metals) and (2) the growth of the equiaxed grains was isotropic, forming spheres ahead of the columnar front. For industrial applications, blades with complex three-dimensional (3-D) 
shapes and defects at various locations must be processed. This implies the treatment of single-crystal substrates with various crystal orientations. As the quality of the repair (i.e., the absence of grain boundaries and cracks) falls with increasing angle between the heat flow direction in the melt pool and the preferred dendrite growth axis, any useful processing map must take this orientation effect into account. Furthermore, the treatment of grain competition in directional heat flow requires the modeling of nonspherical grains.

The objective of the present article is to present, for the first time, microstructure selection maps that show the influence of substrate orientation on the limit of singlecrystal solidification. To simplify the analysis of the complex epitaxial laser metal forming process, laser resolidification (Figure 2) and Bridgman-type directional solidification are considered.

In epitaxial laser metal forming and laser remelting, the specific energy input is relatively low, resulting in small melt pool dimensions and a narrow heat-affected zone. The liquid pool solidifies with high values of temperature gradient $\left(G \sim 10^{5}\right.$ to $\left.10^{6} \mathrm{~K} / \mathrm{m}\right)$, interface velocity $\left(10^{-3}\right.$ to $\left.10^{-1} \mathrm{~m} / \mathrm{s}\right)$, and cooling rate $\left(10^{2}\right.$ to $\left.10^{5} \mathrm{~K} / \mathrm{s}\right)$. Among the

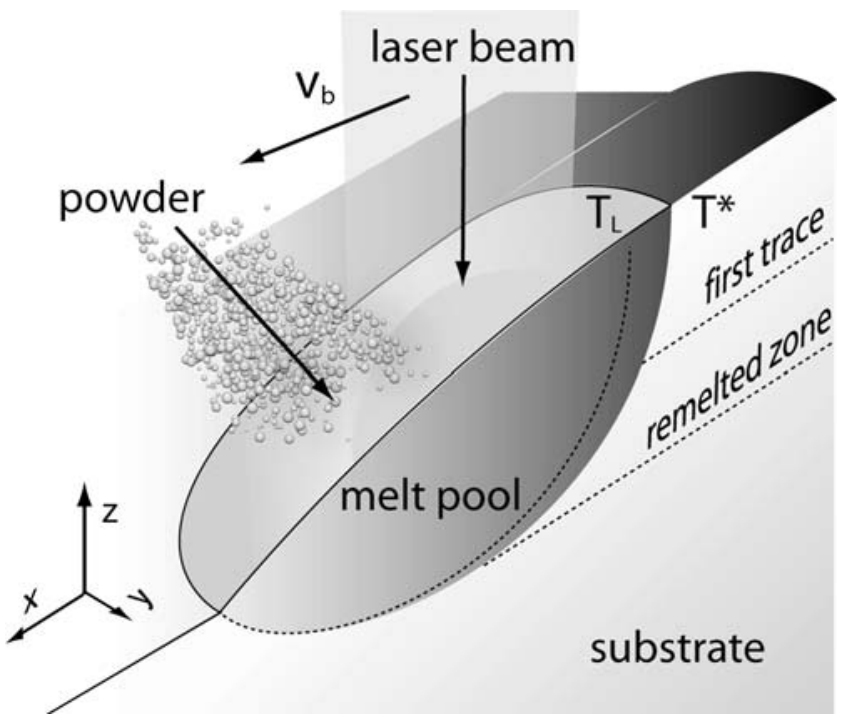

Fig. 1-Schematic representation of the LMF process with lateral supply of powder. View on the surface and on the vertical cut through the center of the trace. The superposition of traces allows the production of various shapes.

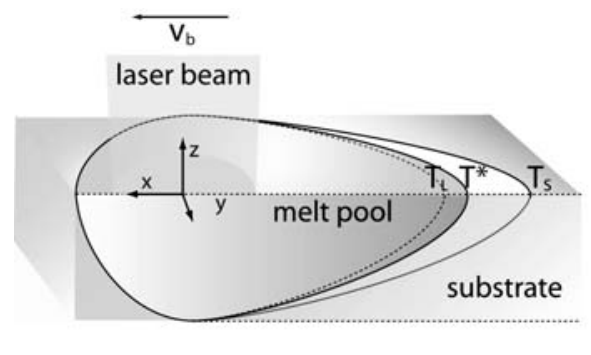

(a) main processing parameters that control the overall process quality are laser power, $P$; scanning velocity, $V_{b}$; laser beam diameter, $D_{b}$; substrate temperature, $T_{0}$; and the mass feeding rate, $\underline{m}$. The laser remelting process (Figure 2) permits the use of analytical models to determine local solidification conditions. Laser remelting may be considered as epitaxial laser metal forming with a zero powder feed rate. ${ }^{[14]}$ It has been used for modeling solidification phenomena encountered during epitaxial laser metal forming and to link the processing parameters with the relevant parameters for microstructure control. ${ }^{[3]}$

In this work, following a brief presentation of the process, typical microstructures obtained in the laser resolidification process and in directional casting of the second generation single-crystal superalloy CMSX-4 will be presented. The microstructures have been observed by the electron backscattered diffraction (EBSD) technique. The phenomena of nucleation and grain growth affecting the development of the grain structure will be discussed, and new results, which are fundamental for full process control, will be given. In particular, the major defect encountered during single-crystal processing is described under the conditions of off-heat flow axis dendritic growth. This is the loss of the crystal orientation of the substrate (loss of epitaxy) through a CET or columnar-to-columnar transition, which is often accompanied by the formation of hot cracks.

\section{EXPERIMENT}

\section{A. Experimental Procedure}

The microstructure development in laser remelting has been examined on cylindrical single-crystal samples of a commercial single-crystal superalloy, CMSX-4. The specimens were machined from directional castings with the [100] crystal orientation within less than $10 \mathrm{deg}$ from the cylinder axis. The samples were fully heat treated (solution treated and aged). In this work, various substrate orientations ranging from $<100>(001)$ to $<100>(011)^{*}$ have been investigated.

*The first index refers to the laser beam scanning direction, always parallel to the specimen axis, and the second refers to the index of the substrate surface on which remelting has been undertaken.

A $1.7 \mathrm{~kW}$ continuous $\mathrm{CO}_{2}$ laser at scanning speeds $1<$ $V_{b}<100 \mathrm{~mm} / \mathrm{s}$ was used for the laser treatment. Experiments were performed on substrates at room temperature. The mean surface temperature of the laser melt pool (inside

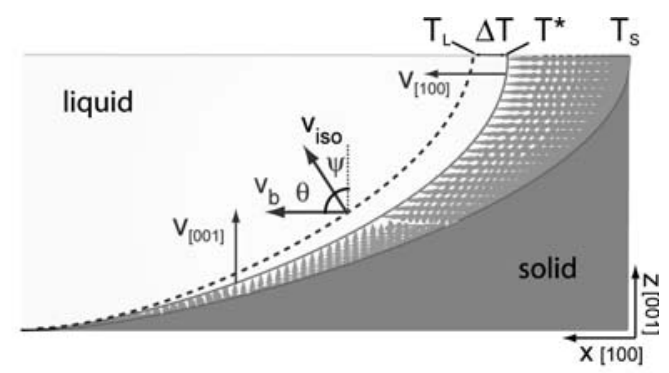

(b)

Fig. 2-Schematic representation of the melt pool during laser remelting. View of the surface and of the vertical cut through the center of the trace. In the experiments, the laser beam velocity $v_{b}$ (along the $x$-axis) was parallel to [100] while the surface normal (z-axis) was oriented between [001] and [011]. 
a 2-mm circle) as a function of process time was recorded for each test by means of a differential optical pyrometer. Only results from steady-state conditions where used. Treatments were carried out under a laminar flow of argon for oxidation protection of the alloy.

Transverse sections of resolidified traces were observed by optical microscopy, after etching in an $\mathrm{HCl}-\mathrm{HNO}_{3}-$ $\mathrm{MoO}_{3}$ solution (Al-rich etching), and analyzed using the EBSD technique. The spatial resolution of this technique was better than $0.2 \mu \mathrm{m}$, while the angular precision of the crystal orientation was less than $0.5 \mathrm{deg}$. The orientation maps were obtained with steps of 1 to $2 \mu \mathrm{m}$.

The nucleation site density was determined by optical microscopy of resolidified specimens at the location of the CET and a randomly uniform nuclei distribution was assumed. ${ }^{[3]}$ It must be emphasized that this technique for measuring the nuclei density depends on the particular processing conditions used and may vary substantially within one melt pool.

\section{B. Experimental Results}

The experiments demonstrated that the crystal orientation of the substrate had a decisive influence on the microstructure development. Depending on process conditions, columnar grains with low- and high-angle grain boundaries and equiaxed grains with high-angle grain boundaries formed during solidification. The limit between the two types of grain was set between 10 and $15 \mathrm{deg} .{ }^{[15]}$ Low-angle grain boundaries remain acceptable for single-crystal repair in current industrial practice. The main microstructural defects encountered during laser remelting of off $<001>$ single crystals were randomly orientated grains at the bottom of the melt pool, i.e., at the very beginning of solidification. As solidification proceeded, these grains competed with each other and with the epitaxial columnar front, often stabilizing a columnar dendritic polycrystal, which often contained grain boundary cracks (hot cracks).

Figure 3(a) shows that laser remelting of a $<100>$ (001) oriented substrate leads to epitaxial growth with a few very small equiaxed grains at the substrate interface, mostly columnar grains with small orientation differences (low-angle grain boundaries) and some high-angle grain boundaries at the surface in the upper right corner. When the substrate surface normal deviated from [001], the volume fraction of misoriented grains increased with the deviation angle. In this case, the heat flow direction at the bottom of the melt pool was no longer aligned with $<001>$. The worst case is shown in Figure 3(b). Here, the microstructure generated in the $<100\rangle(011)$ oriented sample under exactly the same processing conditions as in Figure 3(a) shows a large fraction of columnar grains in the center of the melt pool, which are well aligned with the heat flux but misoriented with respect to the substrate. Many high-angle grain boundaries formed under these conditions, which explains the high susceptibility for hot crack formation (Figure 3(b)).

Similar results were also observed under other conditions. The EBSD images of two samples with different orientations are shown in Figures 4(a) and (b). Figures 4(e) and (f) show micrographs, at higher magnification, of the central regions of Figures 4(c) and (d). At the beginning
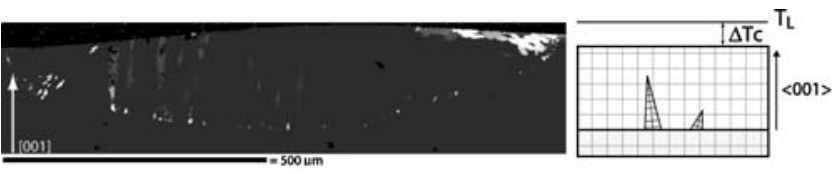

(a)
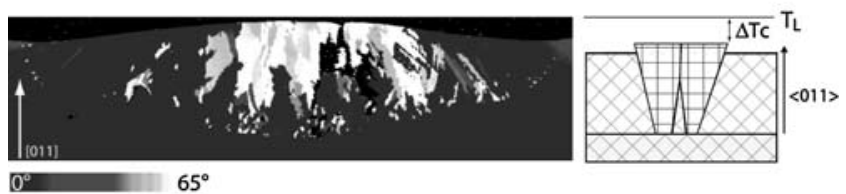

(b)

Fig. 3-Transverse cut of two laser remelting traces (width $=1.5 \mathrm{~mm}$ ) of CMSX-4, produced under the same conditions: $D_{b}=0.76 \mathrm{~mm}, P_{a}=1500$ $\mathrm{W}, v_{b}=100 \mathrm{~mm} / \mathrm{s}$, and $T_{0}=\mathrm{R} T$. Microstructure (EBSD image) for two different substrate orientations: $(a)<100>(001)$ forming a single crystal with some low-angle grain boundaries in the central area of the laser resolidified track, and $(b)<100>(011)$ forming a columnar polycrystal containing hot cracks at the high-angle grain boundaries. The shade of gray represents the misorientation of grains with respect to the main crystallographic orientation of the substrate: dark gray corresponds to zero misorientation, whereas white shows maximum (54.7 deg for cubic systems). Black areas are nonindexed regions corresponding to areas located outside the sample or within cracks and pores. On the right, corresponding representations of the growth competition between the epitaxial crystals and misoriented columnar grains are shown.

of solidification in the center of the melt pool, the solidification morphology was found to be cellular, in the case of the (001) substrate, and dendritic for the (011) substrate. A significant influence of the crystal orientation of the substrate on the microstructural development during laser remelting may be clearly observed. When [001] was perpendicular to the surface, the result in the major part of the laser remelting zone was practically a single crystal (Figures 4(a), (c), and (e)), while for [011] growth, an essentially polycrystalline repair zone was observed through the entire thickness (Figures 4(b), (d), and (f)). It may be concluded that the grains that had a $<100>$ direction closest to the thermal gradient direction had a growth advantage and developed to the detriment of the other grains and the epitaxial solidification front in particular (Figure 3, schematic drawings on the right side).

\section{MODELING}

Figure 2(b) shows the rear part of the melt pool in laser remelting, i.e., the solidification front for a laser beam velocity, $V_{b}$. The interface velocity at the bottom of the pool was zero when the [001] direction was perpendicular to the surface. As has been shown by Rappaz et al. ${ }^{[16]}$ the dendrite tip velocity $V_{h k l}$, the direction of which is given by the substrate orientation $[h k l]$, is generally not equal to the rate of advance of the liquidus isotherm.* The dendrite tip

*Note that the liquidus isotherm $T_{1}$ is not equal to the columnar dendrite tip temperature, as $T_{\text {col }}(\mathrm{z})=T_{1}-\Delta T_{\text {col }}(z)$ (Fig. 2(b)). During FE modeling of laser remelting, however, this effect is neglected.

velocity, $V_{h k l}$, is higher than the velocity normal to the solidification front $V_{\text {iso }}$ by $(\cos \psi)^{-1}$, where $\psi$ is the angle between the preferred dendrite growth axis and the local heat flux direction (Figure 2(b)). 


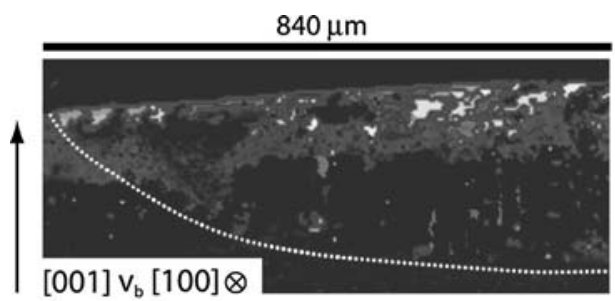

(a)

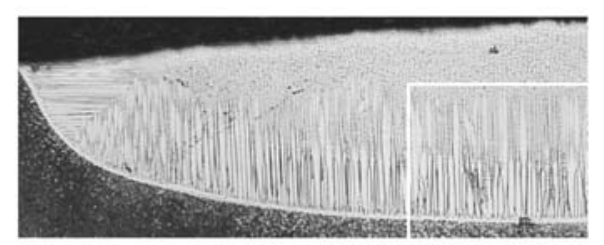

(c)

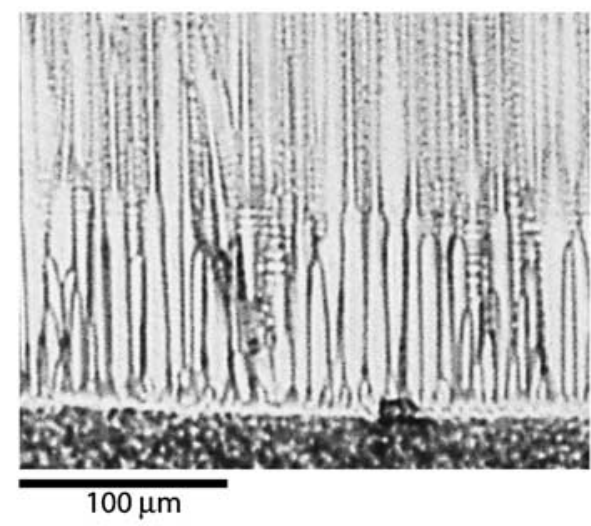

(e)

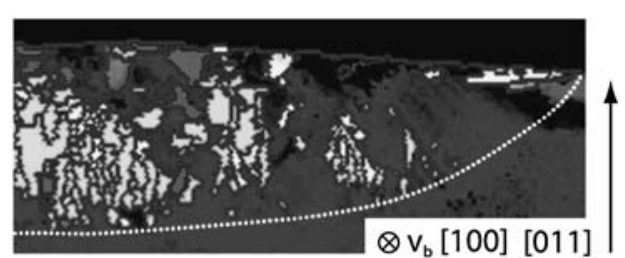

(b)

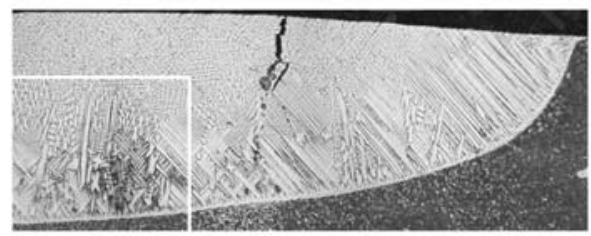

(d)

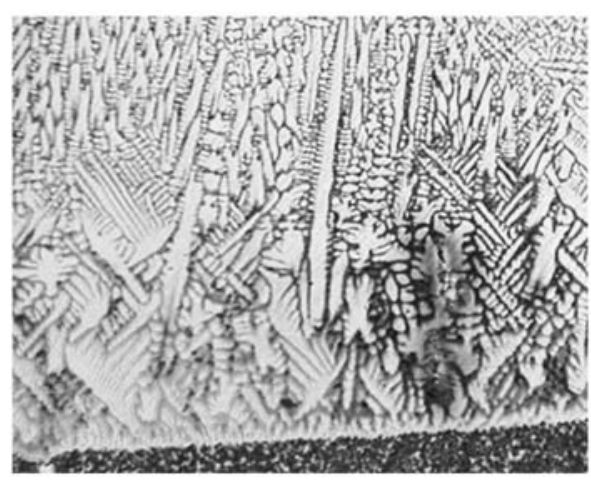

$(f)$

Fig. 4-Transverse sections (EBSD images and optical micrographs) of two remelting traces (CMSX-4, $D_{b}=0.76 \mathrm{~mm}, P_{a}=1700 \mathrm{~W}$, and $\left.\mathrm{v}_{b}=10 \mathrm{~mm} / \mathrm{s}\right)$, for $(a),(c)$, and $(e)<100>(001)$ and $(b),(d)$, and $(f)<100>(011)$ oriented substrates.

\section{A. Analytical Microstructure Modeling of CET for Various Substrate Orientations}

Any high-angle grain boundary above a critical size, which forms in the resolidified material, is detrimental to the properties of the component. Close control of the formation of new grains is essential for the quality of the single-crystal repair. This requires an understanding of the underlying physics of the processes, which will be analyzed subsequently.

The solute field ahead of the columnar dendrites of an alloy leads to a tip undercooling, $\Delta T_{\text {col }}$, and to a constitutionally undercooled zone. ${ }^{[1]}$ The resulting microstructure depends (1) on the local growth conditions $(G, V)$ of the columnar front; (2) on the magnitude of the nucleation undercooling, $\Delta T_{n}$, with respect to dendrite tip undercooling; and (3) on the density of nucleation sites or dendrite fragments, $N_{0}$, in this zone. If the critical undercooling required for nucleation is smaller than the undercooling of the melt, nuclei in the constitutionally undercooled liquid ahead of the columnar front will develop. If their density is high enough, arbitrarily oriented equiaxed grains will form. This phenomenon is known as the CET.

Depending on solidification conditions, three growth regimes are possible: (1) fully columnar growth, where no nucleus is activated; (2) a mixed regime, where a certain volume fraction of equiaxed grains, $\phi_{e}$, coexist with the columnar structure; and (3) a complete transition to equiaxed grains (Figure 5).

According to Hunt, the radius of spherical equiaxed grains is calculated by the integration of the dendrite growth velocity from the nucleation time, $t_{0}$, to the time when the columnar front reaches the grain. ${ }^{[9]}$ It is assumed that for metals, where the Lewis number $(\alpha / D)$ is large, the thermal undercooling of the dendrites is small with respect to the solutal undercooling and columnar and equiaxed dendrites closely follow the same relationship:

$$
\Delta T / \Delta T_{0}=(a \cdot V)^{1 / n}
$$

where $a$ is a material constant, $\Delta T$ is the columnar or equiaxed dendrite tip undercooling, $\Delta T_{0}$ is the equilibrium liquidus solidus interval of the alloy, and $n$ depends on the growth model ( $n=2$ for the simple hemispherical dendrite tip). The resulting mean radius of the equiaxed grains is ${ }^{[9]}$

$$
r_{e}=\int_{t_{0}}^{t} V_{e} \cdot d t=\frac{\Delta T_{\mathrm{col}}^{n+1}}{(n+1) a \Delta T_{0}^{n} \cdot V_{\mathrm{iso}} G} \cdot\left(1-\left[\frac{\Delta T_{n}}{\Delta T_{\mathrm{col}}}\right]^{n+1}\right)
$$




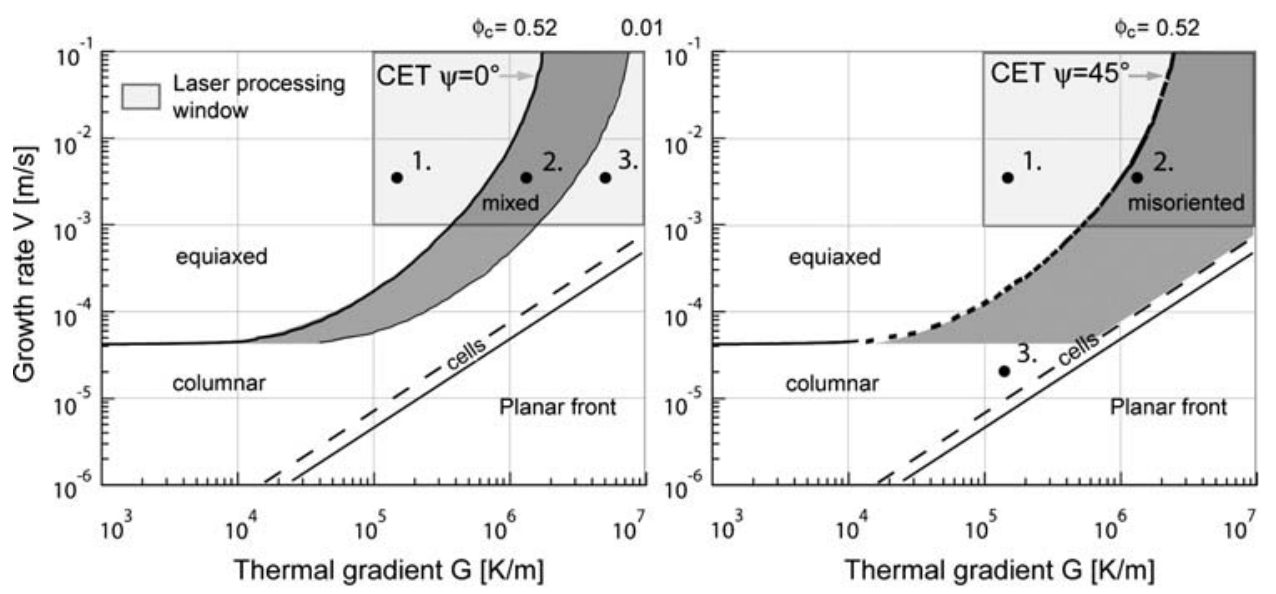

(a)

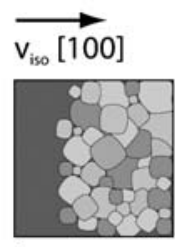

1.

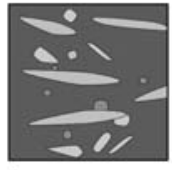

2.

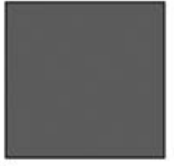

3.

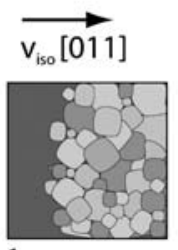

1.

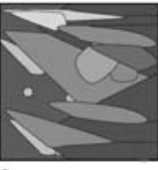

2.

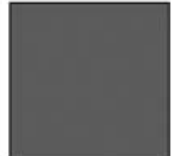

3.

(b)

Fig. 5-(a) Microstructure selection maps for CMSX4 $\left(\Delta T_{n}=2.5 \mathrm{~K}, N_{0}=2 \times 1015\right)$ considering $\psi=0$ deg (left), and off-heat flow axis growth, $\psi=45$ deg (right). Typical solidification conditions for the laser remelting process are indicated by the gray inserts in the upper right corners. (b) Sketches of the corresponding microstructure evolution where both the CET and the OMT transitions are considered. The OMT occurs in the mixed growth regime (b2) when the volume fraction of spurious grains is sufficient to lead to complete overgrowth of the epitaxial solidification front as a result of grain growth competition (b2 right panel).

In the present work, the values of $a$ and $n$ are obtained from the Ivantsov-Marginal Stability (I-MS) dendrite model ${ }^{[18]}$ extended to multicomponent alloys ${ }^{[19,20]}$ and coupled with a thermodynamic database for Ni-base superalloys ${ }^{[21]}$ using ThermoCalc. ${ }^{[22]}$ The physical properties are given in Reference 3. Nucleation has been simplified by assuming that all heterogeneous nucleation sites are activated when the liquid undercooling is greater than a critical nucleation undercooling, $\Delta T_{n}$.

Dendrites of cubic crystals grow along any of the $<100>$ variants, but they preferentially follow the cube direction closest to the heat flux, which means that they grow along the direction with the highest tip temperature or the lowest velocity. This leads to structural transitions between primary dendrite trunks and secondary arms. (Because in a cubic crystal trunks and arms grow orthogonally to each other, this transition is not a CET and the material is still a single crystal.). Taking into account growth along the three cube directions leads to the following expression for columnar dendritic undercooling ${ }^{[16]}$.

$$
\Delta T_{\text {col }}=\Delta T_{0}\left(\frac{a \cdot V_{\text {iso }}}{\cos \psi}\right)^{1 / n}=\Delta T_{0}\left(\frac{a \cdot V_{b} \cdot \cos \theta}{\cos \psi}\right)^{1 / n}
$$

where $\psi$ is the angle between the dendrite growth axis and the local heat flow direction and $\theta$ is the angle between the isotherm normal and the laser scanning direction (Figure 2).
The volume fraction of spherical grains, $\phi_{e q}$, can be obtained using the Johnson-Mehl-Avrami equation:

$$
\phi_{e q}=1-\exp \left(-\frac{4 \pi r_{e}^{3}}{3} \cdot N_{0}\right)
$$

The nucleation site density, $N_{0}$, was determined experimentally, as described in Section II-A.

The limit of columnar growth is given by a critical volume fraction of grains $\phi_{c}$. Under solidification conditions encountered during laser treatment of superalloys usually with high-temperature gradients $\left(>10^{5} \mathrm{~K} / \mathrm{m}\right)$ and relatively high solidification velocities $(>1 \mathrm{~mm} / \mathrm{s})$, the constitutional undercooling of columnar dendrites $\Delta T_{\mathrm{col}}$ is considerably larger than the nucleation undercooling $\Delta T_{n}$ and the volume fraction, $\phi_{e q}$, of equiaxed grains is controlled by nuclei density $N_{0}$ rather than $\Delta T_{n} \cdot{ }^{[3]}$ By neglecting $\Delta T_{n}$, it is found that

$\phi_{e q}=1-\exp \left(-\frac{4 \pi N_{0}}{3} \cdot\left(\frac{a^{1 / n} \Delta T_{0}}{n+1}\right)^{3} \cdot\left(\frac{G^{n}}{V_{\text {iso }}}(\cos \psi)^{n+1}\right)^{-3 / n}\right)$

On this basis, the criterion for columnar growth under laser processing conditions (high $G$ and $V$ ) can be expressed as follows ${ }^{[23]}$ :

$$
\frac{G^{n}}{V_{\text {iso }}}(\cos \psi)^{n+1} \leq K_{\mathrm{CET}}
$$




$$
\text { with } K_{\mathrm{CET}}=a \cdot\left[\left(\frac{-4 \pi N_{0}}{3 \ln \left(1-\phi_{c}\right)}\right)^{1 / 3} \frac{\Delta T_{0}}{n+1}\right]^{n}
$$

where the constant $K_{\mathrm{CET}}$ is a function of alloy parameters and of $\phi_{c}$ (the critical volume fraction of equiaxed grains).

According to a geometric criterion $r_{e}>(1 / 2) N_{0}^{-1 / 3}$ and $\phi_{\mathrm{c}}=0.52$, whereas the limit for columnar growth is reached when $4 / 3 \pi r_{e}{ }^{3} N_{0}<\phi_{c}{ }^{[23]}$ Thus, the critical condition as defined by the left-hand side of Eq. [6] in the melt pool at liquidus temperature depends on crystal orientation and is different from that defined by Hunt. ${ }^{[9]}$ Based on this relationship and taking into account the growth direction of the solidification front with respect to the heat flux direction, the corresponding microstructure map has been determined (Figure 5). Typical solidification conditions for the laser remelting process are indicated in the insert in the upper-right-hand corner.

\section{B. Numerical Modeling of Growth Competition}

For the simulation of the CET and of the grain selection processes during columnar solidification, the so-called CAFE model was used. Rappaz and Gandin ${ }^{[24,25]}$ developed this model using the cellular automaton technique coupled with a finite element method (CAFE) to solve the energy conservation equation. Because the model combines the basic physics of nucleation and dendrite growth (I-MS model $\left.{ }^{[18]}\right)$, it can be used to simulate the complex growth competition between columnar and equiaxed grains (for details of the model, see Appendix I).

In the present analysis, the growth competition between the epitaxial columnar grains and the new grains that appeared in the constitutionally undercooled zone ahead of the columnar growth front was analyzed as a function of both the solidification conditions $(G$ and $V)$ and the crystal orientation of the substrate. Calculations were performed for a cylindrical volume element of directional growth with constant $G$ and $V$, typical for Bridgman experiments (Figure 6). Although these conditions are not the same as in laser remelting, the results of the calculations remain valid for qualitatively describing the mechanism of grain development encountered during laser remelting. In these calculations, the solidification process was initiated on the left of a rod with a given single-crystal orientation (Figure 6(a)) and ended on the right. The remaining epitaxial fraction at any given length could be measured (cross section in Figures 6(b) and (c), where the epitaxial fraction is shown in black). By using this modeling strategy, the influence of $\psi$ on the loss of epitaxy could be defined.

The parameters used for both analytical and numerical calculations of laser processing $(V=1$ to $100 \mathrm{~mm} / \mathrm{s})$ of the Ni-based superalloy CMSX-4 have been evaluated in Ref. 3: the equilibrium liquidus-solidus interval, $\Delta T_{0}=60^{\circ} \mathrm{C}$; the dendrite parameters of Eq. [3], $a=1.2 \mathrm{~s} / \mathrm{m}$ and $n=3.4$; and the value for Eq. [6], $K_{\mathrm{CET}}=2.7 \cdot 10^{24} \mathrm{~K}^{3.4} / \mathrm{m}^{4.4} \mathrm{~s}$. In spite of the relatively low $G$ values and the constant solidification conditions used in these calculations, the results are qualitatively applicable to laser treatment.

\section{Modeling Results}

\section{Analytical model}

Figure 7(a) gives the limit of columnar growth in the usual $G-V$ map calculated for Bridgman solidification conditions. As was observed previously, off-heat flux growth due to crystal anisotropy $(\psi>0 \mathrm{deg})$ extends the equiaxed regime to higher $G$ and lower $V$ values. In this case, i.e., when the heat flux is not collinear with the dendritic growth direction, the dendritic velocity, $V_{h k l}$, is higher than the solidification front velocity, $V_{\text {iso, }}$, and Eq. [6] should be used.

Figure 7(b) illustrates the results of this model graphically, where the orientation of the crystal seed is plotted against the thermal gradient. For a given growth rate $(V=$ $10 \mathrm{~mm} / \mathrm{s}$ ), the three dendrite growth domains defined by the isovolume fraction of equiaxed grains $\left(\phi_{e}=0.52\right.$ and $0.01)$ are given. The equiaxed to mixed regime transition evolves as a function of orientation according to Eq. [6], whereas the mixed to columnar regime remains valid only for $\psi=0 \mathrm{deg}$.

\section{Numerical modeling}

The conditions used for the CAFE calculations were chosen so as to lie within all the domains defined by the analytical CET model (fully columnar, mixed, and equiaxed regimes (Figure 7(a))). Moreover, they were chosen so that the influence of both the thermal gradient and the solidification velocity could be monitored for the various crystal orientations (Figure 7(b)). Three sets of conditions defined by $G=1.5,3$, and $5 \cdot 10^{4} \mathrm{~K} / \mathrm{m}$ were modeled. For each of these sets, the seed orientation was varied between 0 and $45 \mathrm{deg}$. The value of $\Delta T_{n}$ was assumed to be $9 \mathrm{~K}$, while the nucleation site density $N_{0}$ was taken as $3.4 \cdot 10^{10}$ $\mathrm{m}^{-3} \cdot{ }^{[26]}$

The influence of solidification velocity on the microstructure development for a constant thermal gradient $\left(3 \cdot 10^{4} \mathrm{~K} / \mathrm{m}\right)$ and a crystal seed orientation of $\psi=0 \mathrm{deg}$ is shown in Figure 8(a) (the seed crystal orientation is marked in black). The microstructure obtained by the CET model is clearly illustrated here. As the dendrite undercooling increases with $V$, the fraction of new (colored) grains increases and the fully equiaxed regime is reached at the highest velocity, completely superseding the columnar front (compare with Figure 7(a)).

The influence of the thermal gradient is illustrated in Figure $8(\mathrm{~b})$, where the microstructure development is shown for a constant velocity $(V=10 \mathrm{~mm} / \mathrm{s})$ and a [001] axis parallel to the heat flow $(\psi=0 \mathrm{deg})$. Decreasing $G$ leads to an increased volume fraction of equiaxed grains. For $G=1.5 \cdot 10^{4} \mathrm{~K} / \mathrm{m}$, a complete CET was found to be in accordance with the prediction made by the CET model for these specific conditions (Figure 7(a)). The microstructure consists mainly of randomly oriented grains of isotropic morphology, though the influence of the thermal gradient on this morphology can be observed in the aspect ratio of the grains.

The effect of orientation on the resulting microstructure is shown in Figure 8(c) for the conditions defined within the mixed regime $\left(G=5 \cdot 10^{4} \mathrm{~K} / \mathrm{m}, V=10 \mathrm{~mm} / \mathrm{s}\right)$. For a large angle $\psi$, an oriented-to-misoriented transition (OMT) may occur by growth competition; i.e., a mainly columnar 


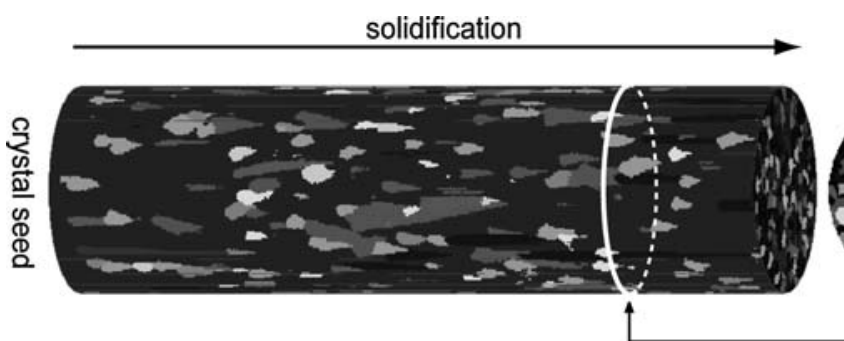

(a)

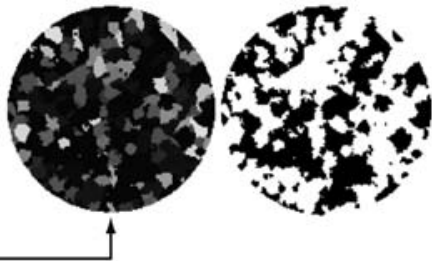

(b)

$(c)$

Fig. 6-(a) Directional solidification (const. $G$ and $V$ ) of a cylindrical volume element simulated by the CAFE model. Solidification starts on the left hand side with a given crystal orientation (black). (b) Transverse sections taken at various distances along the $z$-axis allow the evolution of the epitaxial fraction to be monitored. In $(c)$, the original crystal orientation of section $(b)$ is marked in black.

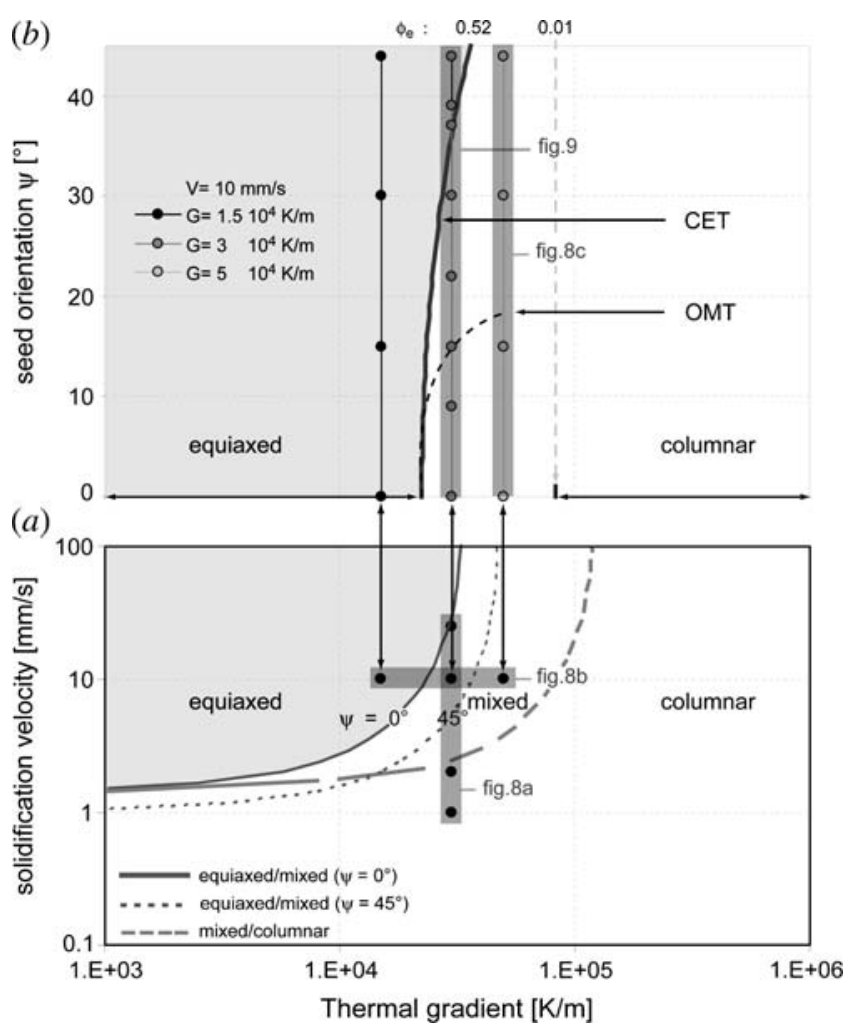

Fig. 7-(a) Microstructure map computed for CMSX-4, $\Delta T_{n}=9 \mathrm{~K}, N_{0}=$ $3.4 \times 10^{10} \mathrm{~m}^{-3}$, and $\phi_{c}=0.49$. In $(b)$, the limit of the equiaxed domain as a function of the angle between the dendritic direction and the heat flux for a constant solidification velocity is shown. The solidification conditions used for the numerical modeling (simulated microstructure evolution using the CAFE model presented in Figs. 8 and 9) are indicated as black points. The limit where residual epitaxial fraction is lost as the solidification proceeds (OMT) is schematically shown in $(b)$ as a dotted line.

structure develops (blue color), which is either a single crystal or a polycrystal. This corresponds to (1) the loss of the initial crystallographic substrate orientation and (2) the formation of at least one transverse grain boundary at the position of the OMT.

Figure 9 shows the calculated evolution of the epitaxial fraction (Appendix II) as a function of the length of the sample for various substrate orientations defined between the two limiting cases: [100] parallel to the cylinder axis, i.e., $\psi=0 \mathrm{deg}$ (top); and [110] parallel to the solidification axis, i.e., $\psi=45 \mathrm{deg}$ (bottom) under the solidification conditions of Figure 7(b). The reduction of $\phi_{\text {epit }}$ is monotonous as the solidification proceeds. In the case of $\psi<15$ deg, a final epitaxial fraction $>0$ is obtained. For $\psi=0$, $\phi_{\text {epit }} \sim 40$ pct, corresponding to a steady-state mixed regime, where the nucleation and growth of spurious grains is well balanced by the growth competition mechanism, i.e., by the continuous elimination of grains less favorably oriented with respect to the heat flux.

For $\psi \geq 15 \mathrm{deg}$, the crystallographic orientation of the substrate was lost completely at various distances. Taking into account the results found for both 3 and $5 \cdot 10^{4} \mathrm{~K} / \mathrm{m}$, the transition between these two solidification regimes is schematically shown in Figure 7(b) as a dotted line (OMT). The epitaxial fraction decreases exponentially; i.e., it decreases at a rate proportional to its value (Figure 9). The remaining epitaxial fraction at a given distance can thus be calculated according to the relationship shown in the figure. Both the decay factor $m$ and the remaining epitaxial fraction $B$ are determined by the angle $\psi$. In these results, the decay factor is actually determined by two components. The first is due to the thermal transient.* The second

*At the very beginning of the calculation, the undercooling ahead of the solidification front remains lower than the value obtained by considering the growth rate of the isotherm imposed. For the conditions used here, this transient regime affects results only over a distance of $0.5 \mathrm{~mm}$. Once steady state is reached, the solidification front grows at the imposed rate and lags behind the isotherm at a distance determined by the growth undercooling.

contribution is due to the growth competition between nucleated misoriented grains and the epitaxial front. This growth competition influences the results. The larger the deviation of the substrate [001] orientation with respect to the heat flux direction, the greater the probability that new misoriented grains will form.

\section{DISCUSSION}

The suppression of grain growth ahead of the columnar front is a required condition for successful single-crystal growth. It is demonstrated here that for certain crystal orientations of the substrate, a small fraction of new grains is sufficient to change the subsequent microstructure completely by the formation of either an equiaxed microstucture or a misoriented columnar structure. 


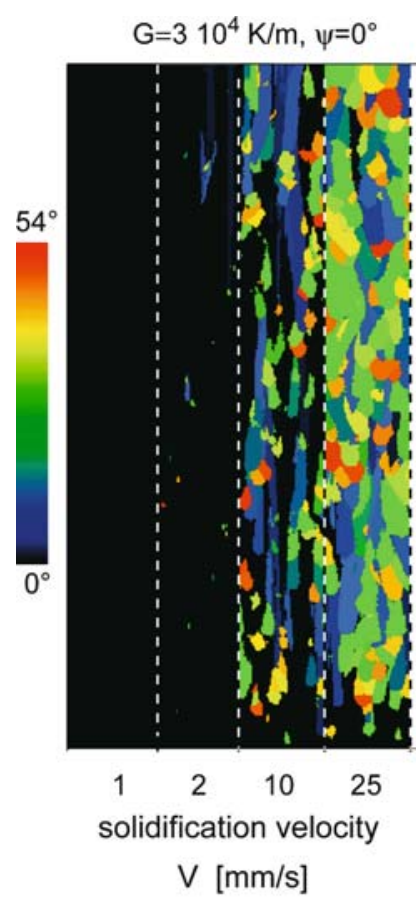

(a)

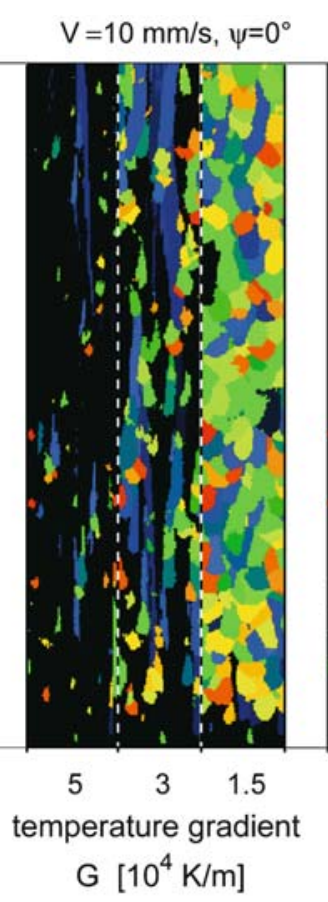

(b)

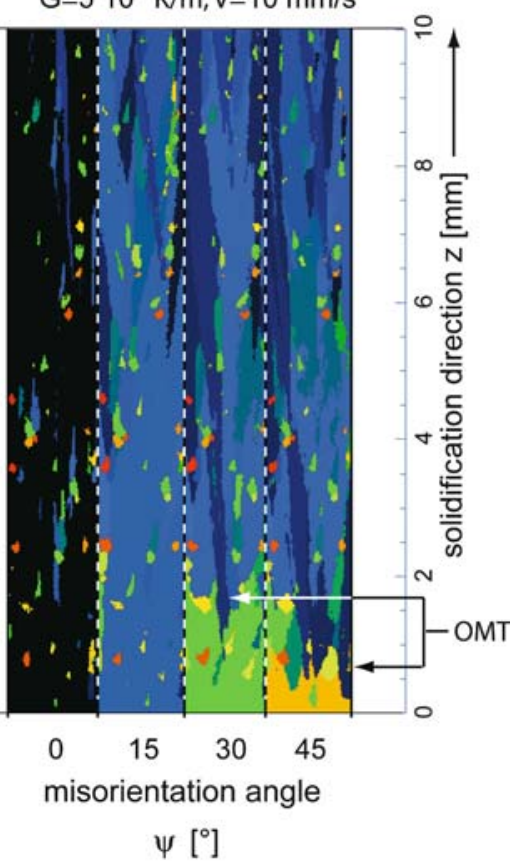

(c)

Fig. 8-Longitudinal sections of directionally solidified specimens (CAFE model) showing microstructure selection as a function of (a) the solidification velocity $(G, \psi$ const.), (b) the temperature gradient ( $V, \psi$ const.), and $(c)$ the seed orientation $(\psi)(G, V$ const.). The orientation color of the seed crystal is black and the deviations in the crystal orientation are marked in blue, green, yellow, and red.

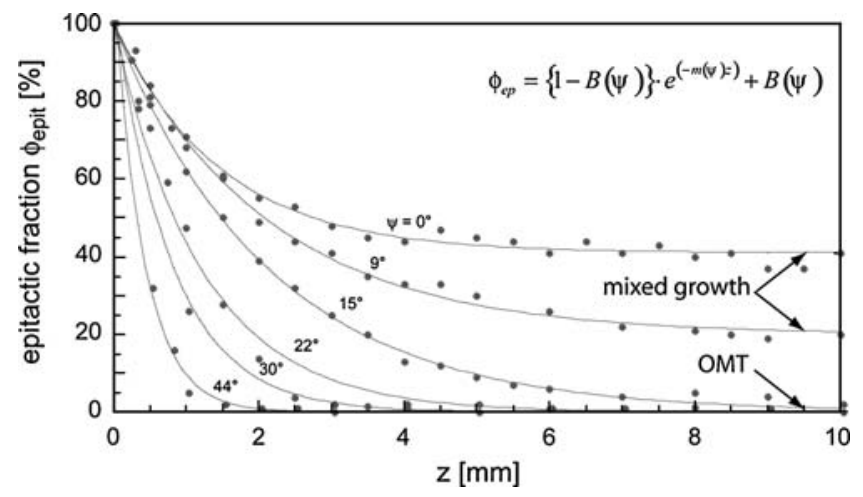

Fig. 9-Calculated evolution of the epitaxial volume fraction of grains as a function of the length of the sample for various angles. The exponential decay fitted to the calculated values are shown $\left(\mathrm{CMSX}-4, \Delta T_{\mathrm{n}}=9 \mathrm{~K}\right.$, $N_{0}=3.4 \times 10^{10} \mathrm{~m}^{-3}, V=10 \mathrm{~mm} / \mathrm{s}$, and $\left.G=3 \times 10^{4} \mathrm{~K} / \mathrm{m}\right)$.

The well-known mechanism of the CET, i.e., the competition between equiaxed grains in the constitutionally undercooled region of the columnar zone with these columnar grains, fails, in many instances, to explain the microstructures obtained during laser treatment of single-crystal substrates. This failure is due to the following reasons. (1) Off-heat flow axis columnar dendritic growth extends the equiaxed regime to higher $G$ and lower $V$ values, thereby reducing the processing window for epitaxial columnar growth (Figure 5(a)). (2) In the analytical CET models, the columnar front is assumed to be overgrown by spherical grains. During laser treatment of single-crystal substrates under high-temperature gradients, grains with columnar morphologies develop within the melt pool, leading to a more complex growth competition mechanism between columnar grains with different orientations. The columnar growth of spurious grains leads to an unavoidable discrepancy between the measured "equiaxed grain fraction" or, more correctly, in this specific case, the misoriented grain fraction and that proposed by the model.

Because no analytical model of a transition under conditions of off-heat flow axis growth is currently available, cellular automaton calculations were used in the present research to model the growth competition between grains. A grain growing closer to the heat flow direction overgrows other grains as a result of the higher tip temperature, which, in a positive temperature gradient, results in a leading position. Another important aspect of the growth competition concerns the branching of secondary and tertiary arms. This sets out the position of the interface between two neighboring grains growing away from each other. In modeling this competition, particular care must be taken in the design of parameters such as the size of the CA cells and the maximum value of the time-step used for the integration of the growth kinetics ${ }^{[27]}$ in order to reasonably reproduce the branching mechanism of dendrite side arms. ${ }^{[28]}$

The results obtained using this model correctly reproduced the prediction of the analytical model proposed by Hunt for $\psi=0$ (Figure 7). For off-heat flow-axis solidification, however, it showed that the microstructure is the consequence of a competition between oriented (epitaxial with the substrate) and misoriented columnar grains leading to a strong reduction of the epitaxial fraction (Figures 8 and 9). From the experimental results, the loss of epitaxy encountered during laser remelting can be treated as a 
competition between the epitaxial grains and misoriented spurious grains. Depending on the orientation $\psi$, the resulting microstructure will lie in between either a small fraction of isolated small grains near the interface substrate/resolidified material, which are overgrown by more favorably oriented grains (Figure 3(a)), or a large proportion of incorrectly textured grains (Figure 3(b)). Even one single equiaxed grain, which develops ahead of the solidification front, will grow at a higher temperature than the epitaxial interface if the growth axis is closer to the heat flow direction. This leads gradually to a complete change of grain orientation and to the formation of detrimental transverse high-angle grain boundaries. Under these conditions, the loss of epitaxy is no longer a function of the volume fraction of equiaxed grains, but is rather dependent on the growth orientation and on the supply of one or more nuclei, possibly through the fragmentation of dendrite branches. The new microstructure is not equiaxed polycrystalline but columnar polycrystalline.

The analytical model for the CET is thus useful only so long as [001] growth parallel to the heat flow is studied. However, due to the 3-D shape of the melt pool, even for the case of [100](001) growth, the primary trunks of the dendrites are aligned with the thermal gradient only within certain regions (bottom and top). As the angle $\psi$ increases along the solidification interface, the aligned dendrites do not grow in the direction of maximum heat flow but continue to grow along the established [001] direction (Figure 2(b)). At $45 \mathrm{deg}$, the situation changes and the dendrites no longer grow perpendicular to, but parallel to, the substrate surface (e.g., along [100]). For a substrate orientation $[100](011)$, the solidification interface predominantly grows off-axis with respect to the heat flow direction. At the bottom of the melt pool, the angle approaches $45 \mathrm{deg}$ and well-developed secondary dendrite arms occur as a result of an equivalent driving force for the growth of primary stems and secondary arms in that region.

Another important aspect that arises during the laser treatment of Ni-based superalloys is due to their susceptibility to solidification cracking. A study of the effect of grain boundary angle on the hot cracking tendency showed that even in alloys of high cracking sensitivity, solidification cracks may be avoided when only sub-boundaries are present. ${ }^{[29]}$ The reason for the increased sensitivity of highangle grain boundaries to solidification cracking seems to be the low coalescence temperature. This leads to an increase of the length of the vulnerable region of the mushy zone and localizes the strain at the boundaries. ${ }^{[30]}$ This explains why completely crack-free laser metal forming and welding of single-crystal turbine blades is possible if the product is a single crystal or contains only subgrain boundaries (Figures 3(a), 3(b), 4(c), and 4(d)). This observation shows the importance of avoiding the formation of high-angle grain boundaries during the solidification process.

\section{CONCLUSIONS}

In previous work on single-crystal repair, the processing window was obtained by the use of the standard CET theory. This model is restricted to [001] columnar dendritic growth parallel to the heat flux and to the isotropic growth of spherical equiaxed grains. In this work, it has been shown by experiment and modeling that in off-heat flow direction dendritic growth, two effects modify the results of the simple CET model.

(1) In off-heat flow axis dendrite growth, the growth rate and therefore the tip undercooling are increased. This extends the regime of equiaxed growth in the $G-V$ space and limits the processing window.

(2) The larger the angle of [001] with respect to the heat flow direction, the larger the probability that grains formed ahead of the columnar zone with their growth axis closer to the heat flow direction will be favored (highest tip temperature criterion).

The new feature of the present research is that in a large temperature gradient, the equiaxed grains are elongated and can overgrow the epitaxial front, thus forming a polycrystalline columnar zone. The single crystal thereby becomes a columnar dendritic polycrystal containing transverse grain boundaries in the transition region. The growth competition during solidification leads to an "oriented columnar to misoriented columnar dendrite transition," called OMT. Transverse high-angle grain boundaries substantially deteriorate the high-temperature properties of the single-crystal alloy and are hot cracking sensitive; they must therefore be avoided.

The mechanisms described here together with other features affecting microstructure development ${ }^{[31]}$ must be taken into account in order to obtain defect-free processing, and thereby the high-quality repair of single-crystal turbine blades.

\section{ACKNOWLEDGMENTS}

The important contributions to this work, in the laser experiments of J.-D. Wagnière (EPFL) and in the calculation of the thermodynamic properties by A. Schnell (Alstom Power), are gratefully acknowledged. This work has been performed within the framework of EU Project No. AWFORS (advanced welding technologies for the repair and salvage of high value engine components of nickel- and titanium-based alloys), sponsored by the Swiss Office for Education and Science, Bern.

Project partners were as follows: Rolls-Royce Deutschland $\mathrm{GmbH}$, Turbomeca SA, ELASIS Sistema Ricerca Nel Mezzogiorno ScpA, Sulzer Innotec AG, RheinischWestfaelische Technische Hochschule Aachen, ENEA Ente per le Nuove Tecnologie, l'Energia e l'Ambiente, CLFA Groupement d'étude et de recherche pour les applications industrielles des laser de puissance, Universita degli Studi di Lecce, and MTU Motoren- und Turbinen-Union Muenchen $\mathrm{GmbH}$.

\section{APPENDIX I}

A 3-D CA algorithm is applied to a regular network of cubic cells fitted to a domain with a given geometry. ${ }^{[24,25]}$ The state assigned to the cells is evaluated as nucleation and growth proceed during solidification. As nucleation proceeds, some cells solidify and their index is changed to a randomly chosen positive integer representing the crystallographic orientation of the grain. This orientation is 
given by three Euler angles, and classes are defined in respect of a random orientation for the newly formed nucleus. The coupling of the $\mathrm{CA}$ and FE algorithms is performed so that the temperature of the center of a CA cell is obtained through linear interpolation of the temperatures calculated at the nodes of the FE grid for each time-step. The time-steps used in the two calculations are different. For the heat diffusion equation, the time-step is large (macro-time-step), in comparison to the CA time-step (micro-time-step) defined in order to limit the propagation of the growth front, i.e., to avoid the capture of several cells per step of time. Nucleation and growth of the grains are then calculated using the CA algorithm. The variation of enthalpy in the cells, due to the contributions of specific heat and latent heat, is then converted into a variation of enthalpy at FE nodes. The dendritic grain envelope is represented by an octahedron. The liquid composition does not vary outside the grain envelope. Within the grain, the solid fraction is obtained by the Scheil microsegregation model. The overall solid fraction and its variation with time and position can thus be calculated. In this approach, the nucleation undercooling and nucleation site density must be specified. For the dendrite growth kinetics, the multicomponent I-MS model ${ }^{[18]}$ was used. The evolution of the grain envelope is calculated as a function of the local nucleation and growth conditions. In order to correct the anisotropy introduced by the cell lattice, an off-centered octahedron growth algorithm is used; ${ }^{[32]}$ moreover, in order to correctly predict the growth competition of two neighboring columnar grains, a parameter ${ }^{[27]}$ is introduced in the selection of the micro-time-steps used for the dendrite tip undercooling determination.

\section{APPENDIX II}

To analyze further the impact of the growth competition mechanism on the microstructure development, the reduction of the epitaxial fraction as a function of the distance was assessed (Figure 9). The evolution of the remaining epitaxial fraction was measured on transverse sections along the cylinder axis. The epitaxial fraction was defined as the proportion of the surface occupied by crystals of the same crystallographic orientation as the seed (Figure 6(c)). This procedure did not measure the fraction of grains nucleated at a given time, as nucleated grains remained present over a certain distance depending on the relative crystallographic orientation with respect to the heat flux direction. In order to compare the results obtained by this modeling strategy to the equiaxed grain volume fraction predicted by the Hunt model, the volume fraction of equiaxed grains predicted by this model must be converted into an area fraction. In the Hunt model, the grains are considered as spherical and nucleate at a given distance from the solidification front. The extended surface fraction, i.e., the surface fraction calculated without taking into account grain impingement, is given by

$$
\phi_{e}^{S}=\pi r_{e}^{2} \cdot N_{S}
$$

where $N_{S}$ is the surface nucleation site density. For a given volume fraction of equiaxed grains, the mean radius was calculated by considering that nuclei are randomly dispersed and by rearranging Avrami's equation:

$$
r_{e}=\left[\frac{-3 \cdot \ln \left(1-\phi^{V}\right)}{4 \pi N_{V}}\right]^{1 / 3}
$$

By converting the nucleation site density per unit volume into the surface nucleation site density,

$$
N_{S}=\left(N_{V} \sqrt{\frac{6}{\pi}}\right)^{2 / 3}
$$

the extended surface fraction could be calculated as follows:

$$
\phi_{e}^{S}=\pi \cdot\left[\frac{-3 \cdot \ln \left(1-\phi^{V}\right)}{4 \pi}\right]^{2 / 3} \cdot\left(\frac{6}{\pi}\right)^{1 / 3}
$$

\section{REFERENCES}

1. M. Gäumann, P.-H. Jouneau, J.-D. Wagnière, and W. Kurz: Proc. Laser Assisted Net Shape Engineering, LANE'97, M. Geiger and F. Vollertsen, eds., Meisenbach, Bamberg, 1997, pp. 651-58.

2. M. Gäumann, S. Henry, F. Cléton, J.-D. Wagnière, and W. Kurz: Mater. Sci. Eng., 1999, vol. 271, pp. 232-41.

3. M. Gäumann, C. Bezençon, P. Canalis, and W. Kurz: Acta Mater., 2001, vol. 49, pp. 1051-62.

4. S. Mokadem, C. Bezençon, J.-M. Drezet, A. Jacot, J.-D. Wagnière, and W. Kurz: Solidification Processes and Microstructures: a Symp. in Honor of Wilfried Kurz, M. Rappaz, C. Beckermann, and R. Trivedi, eds., TMS, Warrendale, PA, 2004, pp. 67-75.

5. C. Bezençon, J.-D. Wagnière, M. Höbel, A. Schnell, M. Konter, and W. Kurz: Proc. Materials for Advanced Power Engineering, 7th COST Conf., Jülich, 2002, J. Lecomte-Beckers, M. Carton, F. Schubert, and P.J. Ennis, eds., Liège, 2002, pp. 503-10.

6. C. Bezençon, A. Schnell, and W. Kurz: Scripta Mater, 2003, vol. 49, pp. 705-09.

7. W. Kurz: Adv. Eng. Mater, 2001, vol. 3, pp. 443-52.

8. M. Gäumann, R. Trivedi, and W. Kurz: Mater. Sci. Eng., 1997, vol. A226 (228), pp. 763-69.

9. J.D. Hunt: Mater. Sci. Eng., 1984, vol. 65, pp. 75-83.

10. S.A. David, J.M. Vitek, S.S. Babu, L.A. Boatner, and R.W. Reed: Sci. Technol. Welding Joining, 1997, vol. 2, pp. 79-88.

11. J.M. Vitek, S.A. David, and L.A. Boatner: Sci. Technol. Welding Joining, 1997, vol. 2, pp. 109-18.

12. J.-W. Park, S.S. Babu, J.M. Vitek, E.A. Kenik, and S.A. David: J. Appl. Phys., 2003, vol. 94, pp. 4203-09.

13. J.M. Vitek: Acta Mater., 2005, vol. 53, pp. 53-67.

14. M. Picasso, C.F. Marsden, J.-D. Wagnière, A. Frenk, and M. Rappaz: Metall. Mater. Trans. B, 1994, vol. 25B, pp. 281-91.

15. D.A. Porter and K.E. Easterling: Phase Transformations in Metals and Alloys, 2nd ed., Chapman and Hall, New York, NY, 1992, p. 118.

16. M. Rappaz, S.A. David, J.M. Vitek, and L.A. Boatner: Metall. Trans. A, 1989, vol. 20A, pp. 1125-38.

17. W. Kurz and D.J. Fisher: Fundamentals of Solidification, 4th ed., Trans Tech Publications, Aedermannsdorf, Switzerland, 1998.

18. W. Kurz, B. Giovanola, and R. Trivedi: Acta Metall., 1986, vol. 34, pp. 823-30.

19. M. Bobadilla, J. Lacaze, and G. Lesoult: J. Cryst. Growth, 1988, vol. 89, pp. 531-44.

20. M. Rappaz and W.J. Boettinger: Acta Mater, 1999, vol. 47, pp. 3205-19.

21. A. Schnell: Alstom Power, Baden, Switzerland, unpublished research, 2004.

22. B. Jansson, M. Schalin, and B. Sundman: J. Phase Equilibria, 1993, vol. 14, pp. 557-62. 
23. C. Bezençon: Ph.D. Thesis, Institute of Materials, Ecole Polytechnique Fédérale de Lausanne, Lausanne, 2002.

24. M. Rappaz and C.-A. Gandin: Acta Metall. Mater, 1993, vol. 41, pp. 345-60.

25. C.-A. Gandin and M. Rappaz: Acta Mater., 1994, vol. 42, pp. 2233-46

26. S. Dobler: Institute of Materials, Ecole Polytechnique Fédérale de Lausanne, Lausanne, unpublished research, 1998.

27. C.-A. Gandin, J.-L. Desbiolles, M. Rappaz, and P. Thévoz: Metall. Mater. Trans. A, 1999, vol. 30, pp. 3153-65.
28. H. Esaka: Ph.D. Thesis, Institute of Materials, Ecole Polytechnique Fédérale de Lausanne, Lausanne, 1986.

29. N. Wang, S. Mokadem, M. Rappaz, and W. Kurz: Acta Mater, 2004, vol. 52, pp. 3173-82.

30. M. Rappaz, A. Jacot, and W.J. Boettinger: Metall. Mater. Trans. B, 2003, vol. 34B, pp. 467-79.

31. S. Mokadem: Ph.D. Thesis, Institute of Materials, Ecole Polytechnique Fédérale de Lausanne, Lausanne, 2004.

32. C.-A. Gandin and M. Rappaz: Acta Mater., 1997, vol. 45, pp. 2187-95. 